\title{
Heavy Flavours in Quark-Gluon Plasma
}

\section{Seyong Kim*}

Department of Physics, Sejong University, Seoul 05000, Republic of Korea

E-mail: skimesejong.ac.kr

\begin{abstract}
Recent progresses in lattice studies of heavy quark and quarkonium at non-zero temperature are discussed. Formulating a tail of spectral functions as a transport coefficient allows lattice determination of momentum diffusion coefficient $(\kappa)$ for charm quark in the heavy quark mass limit and lattice determination of heavy quark/heavy anti-quark chemical equilibration rate in NRQCD. Quenched lattice study on a large volume gives $\kappa / T^{3}=1.8 \cdots 3.4$ in the continuum limit. A recent study with $N_{f}=2+1$ configurations estimates the charmonium chemical equilibration rate $\left(\Gamma_{\text {chem }}\right)$ at $T=400 \mathrm{MeV}$ with $M \sim 1.5 \mathrm{GeV}, \Gamma_{\text {chem }}^{-1} \sim 150 \mathrm{fm} / \mathrm{c}$. Earlier results from the two studies (with different lattice setups and with different Bayesian priors) which calculate bottomonium correlators using NRQCD and employ Bayesian method to calculate spectral functions are summarized: $\Upsilon(1 S)$ survives upto $T \sim 1.9 T_{c}$ and excited states of $\Upsilon$ are sequentially suppressed. The spectral functions of $\chi_{b 1}$ channel shows a Bayesian prior dependence of its thermal behavior: the $\chi_{b 1}$ spectral function with MEM prior shows melting above $T_{c}$ but that with a new Bayesian prior hints survival of $\chi_{b 1}$ upto $\sim 1.6 T_{c}$. Preliminary results from the efforts to understand the difference in the behavior of $\chi_{b 1}$ spectral function are given.
\end{abstract}

34th annual International Symposium on Lattice Field Theory

24-30 July 2016

University of Southampton, UK

\footnotetext{
* Speaker.
} 


\section{Introduction}

Understanding the properties of Quark-Gluon Plasma (QGP) and the transition from the hadronic gas phase to the QGP phase quantitatively is one of the most important subjects in the studies of QCD thermodynamics. Heavy flavours (charm and bottom quark) open an important window for the lattice community to this question together with accompanying difficulties.

Experimentally, investigations of QGP properties usually require comparisons between results of proton-proton collisions and those of relativistic heavy ion collisions (see e.g., the right figure in Fig. 1 where $\Upsilon$ production in $p-p$ collisions is compared with that in $P b-P b$ collisions) and having better grasps on the baseline properties of $p-p$ collisions help us to better distinguish the differences between quarkonium in non-interacting collections of hadron-hadron collisions and that in nucleus-nucleus collisions. In this regard, hadronic processes which involve heavy quark(s) can be advantageous since these processes can be understood more precisely due to "factorization" of the corresponding processes in terms of the short distance perturbative interactions and the long distance non-perturbative matrix elements through effective field theories such as Non-Relativistic QCD [3], potential NRQCD [4]. The same effective field theories can be applied in non-zero temperature environment as long as $T / M<<1$.

Also, as illustrated by the recent measurement of large elliptic flow of charmed meson (the left figure in Fig. 1) from ALICE collaboration, behaviors of heavy quarks in relativistic heavy ion collisions which may be viewed as "a heavy quark in thermal medium" have more than a few surprises and raise many interesting questions for their own sakes. Another effective theory, Heavy Quark Effective Theory (HQET) is useful, where heavy meson (a heavy quark-light quark bound state) can be understood as "brown mug" surrounding a heavy quark [5] due to the heavy quark mass scale. Recently, heavy quark mass limit in non-zero temperature setup allowed nonperturbative formulation of otherwise difficult real time quantities in non-zero temperature $[6,7,8$, 9].
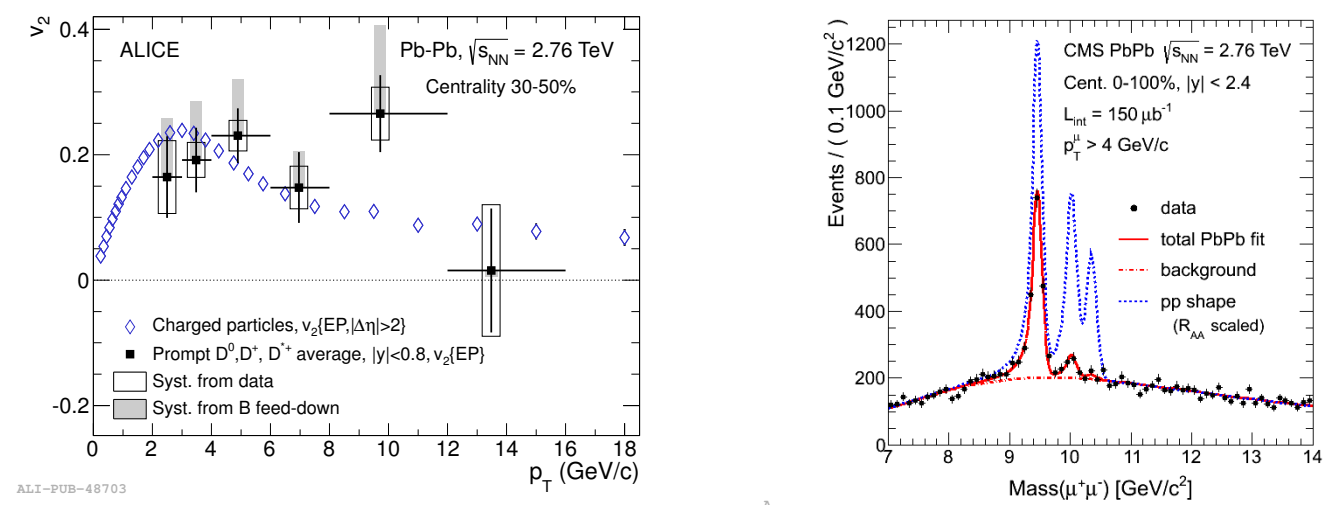

Figure 1: Elliptic flow $\left(v_{2}\right)$ of light charged hadron and charmed meson $\left(D^{0}, D^{+}, D^{*+}\right)$ from ALICE collaboration [1] (left) and sequential suppression of $\Upsilon(1 S), \Upsilon(2 S)$ and $\Upsilon(3 S)$ from CMS collaboration [2].

Of course, relating experiments which includes complex time evolution of highly relativistic scattering of nuclei to the quantities which lattice gauge theory can calculate is highly nontrivial and is involved since lattice gauge theory mostly describes physics in thermal equilibrium. Further- 
more, studying properties of heavy quarks and quarkonia at $T \neq 0$ temperature on a lattice faces many challenges not only because simulations with dynamical charm quark just become possible and simulations with dynamical bottom quark is not possible yet: spectral behaviors of the correlators at non-zero temperature are far more complicated than those at zero temperature due to thermal broadening, transport phenomena, disappearance of bound states, and etc. On the other hand, in isotropic lattice formulation, the temporal correlators at $T \neq 0$ typically have less number of temporal lattice sites than those at zero temperature. Deducing more information from less data points is unavoidable.

Here, we discuss heavy quarks and quarkonia at $T \neq 0$ focusing on (1) two real time quantities concerned with heavy quark kinetic/chemical equilibriation and (2) in-medium quarkonium studied with NRQCD correlators and Bayesian reconstructions of corresponding spectral functions. Although in this conference, a talk on "open charm on anisotropic lattice" [10], a talk on new attempt on the inverse integral transform method together with a new determination of charm diffusion constant [11], another talk on new determination of the charmonium diffusion constant [12], a talk on new stochastic method for reconstructing spectral function [13], and a talk on the Debye screening mass of the potential determined by first principles method [14] are given and, due to lack of space, these are not covered by this plenary talk.

\section{Heavy Quarks}

Hydrodynamic flow of charmed meson in relativistic $\mathrm{Pb}-\mathrm{Pb}$ collisions is comparable to that of light hadron (Fig. 1), which suggests that an effective thermalization of charm quark is similar to that of light quarks [1]. This is in conflict with a perturbative consideration and calls for a lattice QCD determination of transport properties of heavy quarks in QGP. Transport properties are nonequilibrium characteristics and are usually accessed by the "transport peak" of the spectral function for the corresponding correlators in thermal equilibrium through Kubo relations [15]. However, obtaining the transport coefficient directly from the "transport peak" of the spectral function for heavy quark current-current correlator is hard because the width of transport peak in the spectral function scales as $\sim \alpha_{s}^{2} T^{2} / M$ and the peak is narrow for the heavy quark case. A HQET based formulation by [6] allows one to extract a transport coefficient from the "power-law frequency tail" of the spectral function, instead of "zero frequency limit peak" of the spectral function. A quenched lattice determination of the transport coefficient related to the kinetic equilibriation of heavy quark in thermal environment following this formulation is reported recently in [16]

One of other interesting questions concerning the behavior of heavy quarks in QGP is whether the abundance of heavy quarks/anti-heavy quarks in QGP follows thermal Boltzmann distribution. Experimentally, it is related to whether the chemical equilibriation rate of heavy quarks is large compared to the lifetime of QGP achieved in relativistic heavy ion collisions. Similarly to the case of kinetic equilibriation of heavy quark in thermal medium [6], chemical equilibriation of heavy quark/heavy anti-quark in thermal equilibrium can be nonperturbatively defined through densitydensity correlators in NRQCD limit [7,8]. A lattice determination (in full $N_{f}=2+1$ dynamical simulation) of the chemical equilibriation rate of heavy quark/anti-heavy quark is reported for the first time [9]. 


\subsection{Kinetic equilibriation}

Let us briefly describe the extraction of a transport coefficient from the power-law tail of a spectral function. The hydrodynamic properties of a heavy quark moving through a thermal medium is characterized by the diffusion coefficient, $D$, defined as

$$
D=\frac{1}{3 \chi_{00}} \lim _{\omega \rightarrow 0} \sum_{i=1, . .3} \frac{\rho_{V}^{i i}(\omega)}{\omega}, \quad \rho_{V}^{i i}(\omega)=\int_{-\infty}^{\infty} d t e^{i \omega t} \int d^{3} x\left\langle\frac{1}{2}\left[J^{i}(t, \mathbf{x}), J^{i}(0, \mathbf{0})\right]\right\rangle
$$

where $J^{i}=\bar{\psi} \gamma^{i} \psi$ ( $\psi$ is the relativistic heavy quark field) through fluctuation-dissipation theorem. $\chi_{00}$ is a susceptibility related to the 0 -th component of the four-current by

$$
\chi_{00}=\frac{1}{T} \int d^{3} x\left\langle J^{0}(t, \mathbf{x}) J^{0}(t, \mathbf{0})\right\rangle .
$$

The transport peak, $\omega \rightarrow 0$ limit of $\rho_{V}^{i i}(\omega)$, becomes narrower as $M \rightarrow \infty$ and is difficult to access. Instead, from the observation that in a large heavy quark mass limit, the momentum diffusion $\operatorname{constant}(\kappa)$, the drag coefficient $\left(\eta_{D}\right)$ and the kinetic equilibriation time $\left(\tau_{\text {kin }}\right)$ are related to $D$ by

$$
D=\frac{2 T^{2}}{\kappa}, \quad \eta_{D}=\frac{\kappa}{2 M T}, \quad \tau_{\text {kin }}=\frac{1}{\eta_{D}}
$$

with an assumption of "narrow transport peak" and a Lorentzian width around the peak [6], authors of [16] focus on the momentum diffusion coefficient, $\kappa$. The mass dependent momentum diffusion coefficient, $\kappa^{M}$, is defined as

$$
\kappa^{M}=\left.\frac{M_{\mathrm{kin}}^{2} \omega^{2}}{3 T \chi^{00}} \sum_{i} \frac{2 T \rho_{V}^{i i}(\omega)}{\omega}\right|_{\eta_{D}<<|\omega| \leq \omega_{U V}},
$$

where $M_{\text {kin }}$ is the kinetic mass of heavy quark and $\omega_{U V}$ is a cut-off isolating the narrow transport peak. It can then be written as

$$
\kappa=\frac{1}{3 T} \sum_{i=1,3} \lim _{M \rightarrow \infty} \frac{1}{\chi_{00}} \int d t d \mathbf{x}\left\langle\frac{1}{2}\left(\left[\phi^{\dagger} g E^{i} \phi-\theta^{\dagger} g E^{i} \theta\right](t, \mathbf{x}),\left[\phi^{\dagger} g E^{i} \boldsymbol{\phi}-\theta^{\dagger} g E^{i} \theta\right](0, \mathbf{0})\right)\right\rangle,
$$

where $\phi$ is the non-relativistic spinor for heavy quark, $\theta$ is that for heavy anti-quark, and $E^{i}$ is the color electric field. One can express Eq. 2.5 as

$$
G_{E}(\tau)=-\frac{1}{3} \sum_{1=1,3} \frac{\left\langle\operatorname{Re} \operatorname{Tr} U(\beta, \tau) g E_{i}(\tau, \mathbf{0}) U(\tau, 0) g E_{i}(0, \mathbf{0})\right\rangle}{\langle\operatorname{Re} \operatorname{Tr}[U(\beta, 0)]\rangle},
$$

where $U$ is the product of the time directional links. This is amenable to a lattice calculation although it is still a difficult lattice measurement because the observable is gluonic and suffers from large statistical noise. Overcoming this difficulty, authors of [16] announced result of their multiyear effort. On large quenched lattices $\left(64^{3} \times 16 \sim 192^{3} \times 48\right)$, at a fixed temperature $\left(T=1.5 T_{c}\right)$, they performed a continuum extrapolation of lattice-measured correlators (Eq. 2.6) obtained with a multi-level (actually two-level) algorithm as the first step. Then, instead of applying a general Bayesian reconstruction of the full spectral function for Eq. 2.6, the IR limit and the UV limit of the spectral function is argued to be a specific functional form. These two limits are interpolated 


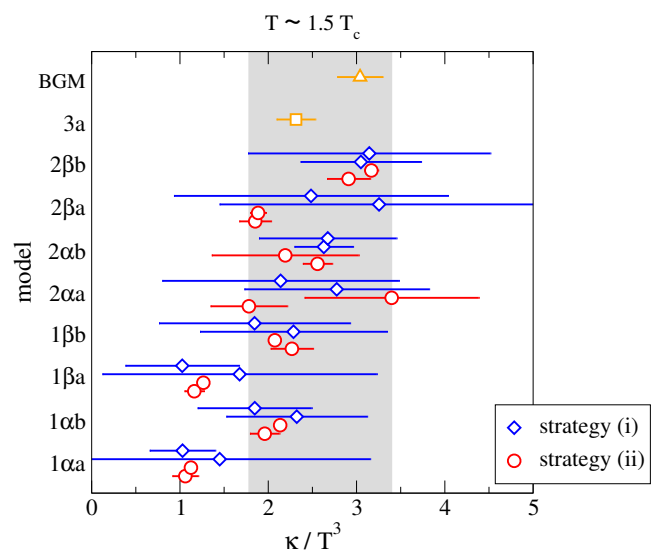

Figure 2: The fitted results from various fitting strategies and fitting forms [16], where the gray band is their final estimate (refer to [16] for the various symbols).

using various fitting forms and strategies. Fitted results are also tested against standard Maximum Entropy Method. Fig. 2 shows the fitted results of $\kappa / T^{3}$. The gray band corresponds to $\kappa / T^{3}=$ $1.8-3.4$, which gives an estimate for the kinetic equilibriation time scale,

$$
\tau_{\text {kin }}=\frac{1}{\eta_{D}}=(1.8 \cdots 3.4)\left(\frac{T_{c}}{T}\right)^{2}\left(\frac{M}{1.5 \mathrm{GeV}}\right) \mathrm{fm} / \mathrm{c} .
$$

This suggests that near $T_{c}$, charm quark kinetic equilibriation is as fast as light parton kinetic equilibriation which is $\sim 1 \mathrm{fm} / \mathrm{c}$.

\subsection{Chemical equilibriation}

Similar to the kinetic equilibriation of heavy quarks in QGP, one can define the chemical equilibriation rate as a transport coefficient from a spectral function of a density-density correlator $[7,8]$. The chemical equilibriation is related to how the number density $(n)$ is adjusted, where

$$
\left(\partial_{t}+3 h\right) n=-c\left(n^{2}-n_{\mathrm{eq}}^{2}\right)
$$

in Boltzmann equation approach and

$$
\left(\partial_{t}+3 h\right) n=-\Gamma_{\text {chem }}\left(n-n_{\text {eq }}\right)+\mathscr{O}\left(n-n_{\text {eq }}\right)^{2}
$$

in a linearized form near the equilibrium where $h$ is a kind of "Hubble expansion constant" if expanding QGP is considered. $\Gamma_{\text {chem }}=2 c n_{\text {eq }}$ is called the chemical equilibriation rate. The linearized form can be described in terms of a Langevin equation

$$
\frac{\partial}{\partial t} \delta n(t)=-\Gamma_{\text {chem }} \delta n(t)+\xi(t), \quad\left\langle\left\langle\xi(t) \xi\left(t^{\prime}\right)\right\rangle\right\rangle=\Omega_{\text {chem }} \delta\left(t-t^{\prime}\right), \quad\langle\langle\xi(t)\rangle\rangle=0
$$

where $\delta n(t)=n-n_{\text {eq }}$ and $\langle\langle\cdots\rangle\rangle$ denotes the average over the Langevin noise. A transport property can be studied through the spectral function of a two-point correlator

$$
\Delta\left(t, t^{\prime}\right)=\left\langle\frac{1}{2}\left\{\delta n(t), \delta n\left(t^{\prime}\right)\right\}\right\rangle
$$


$\Omega_{\text {chem }}$ and $\Gamma_{\text {chem }}$ is related to the tail of the spectral function for $\Delta\left(t, t^{\prime}\right)$,

$$
\Omega_{\text {chem }}=\lim _{\Gamma_{\text {chem }} \ll \omega \ll \omega_{U V}} \omega^{2} \int_{-\infty}^{\infty} d t e^{i \omega\left(t-t^{\prime}\right)}\left\langle\frac{1}{2}\left\{\delta n(t), \delta n\left(t^{\prime}\right)\right\}\right\rangle, \quad \Gamma_{\text {chem }}=\frac{\Omega_{\text {chem }}}{2 \chi_{f} M^{2}},
$$

where $\chi_{f}$ is the quark-number susceptibility related to the heavy flavour. For a lattice calculation of the chemical equilibriation of heavy quark, we need the imaginary-time formulation of Eq. 2.12. Since in non-relativistic limit,

$$
H=M\left(\theta^{\dagger} \theta-\phi^{\dagger} \phi\right)
$$

is related to the heavy quark number operator, the density-density correlator in the imaginary time formalism,

$$
\Delta(\tau)=\int d^{3} \mathbf{x}\langle H(\tau, \mathbf{x}) H(0, \mathbf{0})\rangle, \quad 0<\tau<\frac{1}{T}=\beta,
$$

is considered. Within NRQCD framework, the first order perturbation due to pair annihilation for S-wave $\left(\operatorname{Im} f_{1}\left({ }^{1} S_{0}\right) \theta^{\dagger} \phi \theta \phi^{\dagger}\right)$ gives

$$
\Delta(\tau) \approx \frac{\operatorname{Im} f_{1}\left({ }^{1} S_{0}\right)}{\pi M^{2}} \int d^{3} \mathbf{x} \int d^{3} \mathbf{y} \int_{0}^{\beta} d \tau_{1} \int_{0}^{\beta} d \tau_{2} \frac{\left\langle H(\tau, \mathbf{x}) H(0, \mathbf{0})\left(\boldsymbol{\theta}^{\dagger} \phi\right)\left(\tau_{1}, \mathbf{0}\right)\left(\phi^{\dagger} \boldsymbol{\theta}\left(\tau_{2}, \mathbf{0}\right)\right\rangle\right.}{\left|\tau_{1}-\tau_{2}\right|} .
$$

Then, from the tail of the spectral function,

$\Omega_{\text {chem }}=16 \operatorname{Im} f_{1}\left({ }^{1} S_{0}\right) \frac{1}{Z} \sum_{m, n} e^{-\beta E_{m}}\left\langle q \bar{q} m\left|\theta^{\dagger} \phi\right| n\right\rangle\left\langle n\left|\phi^{\dagger} \theta\right| q \bar{q} m\right\rangle=16 \operatorname{Im} f_{1}\left({ }^{1} S_{0}\right) \frac{1}{Z} \operatorname{Tr}\left[e^{-\beta \mathscr{H}}\left(\boldsymbol{\theta}^{\dagger} \phi\right)\left(0^{+}, \mathbf{0}\right)\left(\boldsymbol{\phi}^{\dagger} \boldsymbol{\theta}\right)(0, \mathbf{0})\right]$,

where $q, \bar{q}$ are heavy quark and heavy anti-quark, and $m, n$ denote the other degrees of freedom. Note that

$\frac{1}{Z} \operatorname{Tr}\left[e^{-\beta \mathscr{H}}\left(\boldsymbol{\theta}^{\dagger} \phi\right)\left(0^{+}, \mathbf{0}\right)\left(\phi^{\dagger} \boldsymbol{\theta}\right)(0, \mathbf{0})\right]=\left\langle\left(\boldsymbol{\theta}^{\dagger} \phi\right)\left(0^{+}, \mathbf{0}\right)\left(\phi^{\dagger} \boldsymbol{\theta}\right)(0, \mathbf{0})\right\rangle=\operatorname{Tr}\left\langle G^{\theta}(\beta, \mathbf{0} ; 0, \mathbf{0}) G^{\theta^{\dagger}}(\beta, \mathbf{0} ; 0, \mathbf{0})\right\rangle$

and

$$
\chi_{f}=\int d^{3} \mathbf{x}\left\langle\left(\theta^{\dagger} \theta+\phi \phi^{\dagger}\right)(\tau, \mathbf{x})\left(\boldsymbol{\theta}^{\dagger} \boldsymbol{\theta}+\phi \phi^{\dagger}\right)(0, \mathbf{0})\right\rangle=2 \operatorname{Re} \operatorname{Tr}\left\langle G^{\theta}(\beta, \mathbf{0} ; 0 \mathbf{0})\right\rangle .
$$

Then, the thermal averaged Sommerfeld factors become

$$
\bar{S}_{1}=\frac{P_{2}}{P_{1}^{2}}, \quad \bar{S}_{8}=\frac{N_{c}^{2} P_{3}-P_{2}}{\left(N_{c}^{2}-1\right) P_{1}^{2}}
$$

with

$$
\begin{gathered}
P_{1}=\frac{1}{2 N_{c}} \operatorname{Re}\left\langle G_{\alpha \alpha ; i i}^{\theta}(\beta, \mathbf{0} ; 0, \mathbf{0})\right\rangle, \quad P_{2}=\frac{1}{2 N_{c}}\left\langle G_{\alpha \sigma ; i j}^{\theta}(\beta, \mathbf{0} ; 0, \mathbf{0}) G_{\sigma \alpha ; j i}^{\theta \dagger}(\beta, \mathbf{0} ; 0, \mathbf{0})\right\rangle, \\
P_{3}=\frac{1}{2 N_{c}^{2}}\left\langle G_{\alpha \alpha ; i j}^{\theta}(\beta, \mathbf{0} ; 0, \mathbf{0}) G_{\sigma \sigma ; j i}^{\theta^{\dagger}}(\beta, \mathbf{0} ; 0, \mathbf{0})\right\rangle
\end{gathered}
$$

for the color singlet channel and the color octet channel respectively. The Sommerfeld factor $(S)$ usually means the enhancement due to the attractive long range interaction between slowly moving, 
annihilating heavy particles (heavy quark and heavy anti-quark for QCD) compared to the Born matrix element for the pair annihilation $\left(\left|\mathscr{M}_{\text {resummed }}\right|^{2}=S\left|\mathscr{M}_{\text {tree }}\right|^{2}\right)$ [17]. Then,

$$
\Gamma_{\text {chem }}^{1}=\frac{\operatorname{Im} f_{1}\left({ }^{1} S_{0}\right)}{M^{2}} \chi_{f} \frac{\bar{S}_{1}}{N_{c}}, \quad \Gamma_{\text {chem }}^{8}=\frac{\operatorname{Im} f_{8}\left({ }^{1} S_{0}\right)}{M^{2}} \chi_{f} \frac{\left(N_{c}^{2}-1\right) \bar{S}_{8}}{2 N_{c}^{2}} .
$$

or if Boltzmann equation is considered [7],

$$
\Gamma_{\text {chem }} \simeq \frac{8 \pi \alpha_{s}^{2}}{3 M^{2}}\left(\frac{M T}{2 \pi}\right)^{3 / 2} e^{-M / T}\left[\frac{\bar{S}_{1}}{3}+\left(\frac{5}{6}+N f\right) \bar{S}_{8}\right]
$$

with the assumption that the octet Sommerfeld factors are spin-independent.

Using FASTSUM configurations [18] $\left(24^{3} \times N_{\tau}, a_{s} / a_{\tau}=3.5 a\right.$-fixed $\left(a_{s}=0.1227(8) \mathrm{fm}\right)$, $N_{f}=2+1$ with $m_{\pi} \simeq 400 \mathrm{MeV}, m_{K} \simeq 500 \mathrm{MeV}$ ), [9] calculated $\bar{S}_{1}$ and $\bar{S}_{8}$ (Fig. 3). A naive perturbative estimate for the singlet Sommerfeld factor, $\bar{S}_{1}$, (the right figure, the red line) $\sim \mathscr{O}(10)$. The lattice value ranges $\sim \mathscr{O}(100-1000)$. Only when a bound state contribution is included in the model spectral function of heavy quark/heavy anti-quark system, the analytic estimate becomes close to the lattice value (the right figure, the black line). For the repulsive octet channel, $\bar{S}_{8}$, both the lattice value and the analytic estimate stays $\sim \mathscr{O}(1)$. The huge increase in the singlet Sommerfeld factor illustrates the importance of non-perturbative effect near $T_{c}$. For a phenomenologically
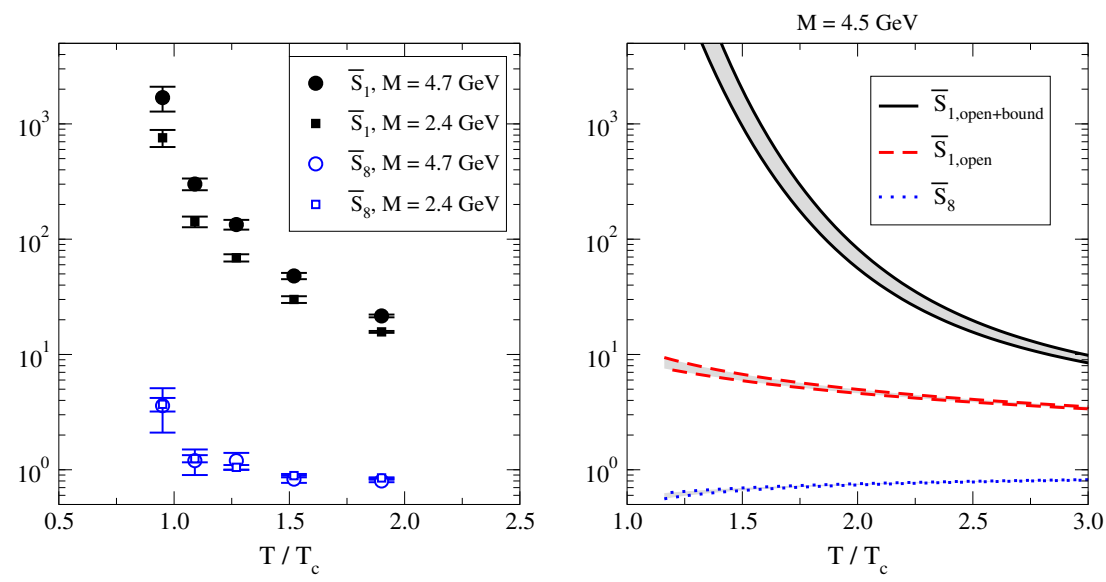

Figure 3: The thermal averaged singlet channel Sommerfeld factor $\left(\bar{S}_{1}\right)$ and octet channel factor $\left(\bar{S}_{8}\right)$ [9] (left). Note that the vertical axis is in the logarithmic scale. The right shows a perturbative calculation of these quantities using a spectral function with a bound state (black) and using a spectral function without a bound state (red) for the singlet channel

interesting case, one can estimate the chemical equilibriation rate for a charm quark ( $M \sim 1.5 \mathrm{GeV})$ using Eq. 2.23 at $T \sim 400 \mathrm{MeV}$, with $\bar{S}_{1} \sim 15, \bar{S}_{8} \sim 0.8$, which is $\Gamma_{\text {chem }}^{-1} \sim 150 \mathrm{fm} / \mathrm{c}$. This suggests that within the QGP phase lifetime $\sim 10 \mathrm{fm} / \mathrm{c}$ of the current relativistic heavy ion collision, charm quark does not chemical equilibrate, as expected.

\section{Quarkonium}

Despite long and intense efforts by the lattice community, a quantitative understanding on whether bound states of heavy quark and heavy anti-quark pair can exist in thermal medium based 
on first principle calculation is still lacking. An early (qualitative) picture of quarkonium "melting" based on a screened potential [19] is too simplistic since there is an imaginary part of potential in thermal medium [20]. The recent directions taken to study "quarkonium in medium" are roughly categorized as,

- (1) calculate the potential between heavy quarks from the spectral function of Wilson loop/Wilson line correlator using $T \neq 0$ gauge fields (see e.g., [21, 22, 23]). Or (2) calculate point-split quarkonium correlators of relativistic heavy quark propagators using $T \neq 0$ gauge fields and define the potential by "HAL QCD method" [24] (e.g, [25, 26]). Then solve the Schrödinger equation for the quarkonium states using the lattice calculated potential [27, 28].

- calculate a fully relativistic heavy quark propagator using $T \neq 0$ gauge fields and obtain quarkonium correlators from this relativistic heavy quark propagator. Then, obtain the spectral function of the temporal quarkonium correlator using Bayesian method [29]. Full spectral features including a transport peak, bound state features and melting of bound states are expected.

- calculate heavy quark propagator using NRQCD under the background of $T \neq 0$ gauge fields and obtain non-relativistic temporal quarkonium correlator. Then, obtain the spectral function of the quarkonium correlators using various Bayesian methods [30, 31, 32, 33, 18, 34]. In NRQCD, absence of a transport feature in the spectral function (since $\sim 2 M$ scale is integrated out) allows us to focus on the binding and melting features of states in the spectral function.

Here we concentrate on the results from lattice NRQCD study of quarkonium in non-zero temperature, i.e., studies of $T \neq 0$ quarkonium correlators $\left(G\left(\tau / a_{\tau}\right), \tau / a_{\tau}=0, \cdots\left(N_{\tau}-1\right), a_{\tau}\right.$ is the temporal lattice spacing) by calculating a heavy quark propagator using lattice NRQCD and obtain the spectral functions of the NRQCD quarkonium correlators using Bayesian method. There are many practical advantages of the NRQCD formalism over full QCD for a lattice study of quarkonium: quarkonium correlators from a heavy quark propagator which is calculated with NRQCD is highly accurate (typically the statistical error is $\sim \mathscr{O}\left(10^{-4}\right)$ ) compared to the quarkonium correlators calculated with relativistic QCD. NRQCD heavy quark propagator can be calculated fast since NRQCD Lagrangian is a first order in time and is numerically an initial value problem which allows a larger $\tau$ than a relativistic propagator. However, the continuum limit of the correlators can not be taken because the spatial lattice spacing must satisfy $M a_{s} \sim \mathscr{O}(1)$.

Given $G(\tau)$,

$$
G(\tau)=\int_{0}^{\infty} \frac{d \omega}{2 \pi} K(\omega, \tau) \rho(\omega) \quad 0 \leq \tau<\frac{1}{T}
$$

where the kernel $K(\tau, \omega)$ becomes $\sim e^{-\omega \tau}$ for NRQCD and $\left(e^{-\omega \tau}+e^{-\omega(1 / T-\tau)}\right) /\left(1-e^{-\omega T}\right)=$ $\cosh (\omega(\tau-2 / T)) / \sinh (\omega 1 / 2 T)$ for full QCD, non-zero temperature behavior is studied by the temperature dependence of the spectral function, $\rho(\omega)$. The integral transform, Eq. 3.1, shows the crux of studying the in-medium property of quarkonium through the spectral function of the Euclidean correlator. Since we do not know the analytic structure of the spectral function, this inverse integral transform problem is ill-posed. To make the numerical problem worse, the number of temporal lattice sites for $G(\tau)$ at $T \neq 0$ is typically smaller than that at zero temperature while the 
spectral structure is expected to be more complicated at $T \neq 0$ than that at zero temperature (i.e., we need to extract more "information" with less "data"). Simplified form of the kernel for NRQCD gives several advantages over the kernel for full QCD: (1)a "constant contribution problem" is absent $[36,35,37]$. (2) $\tau / a_{\tau}$ of $G\left(\tau / a_{\tau}\right)$ can extend its range to $1 / T a_{\tau}-1$. (3)the inverse integral transform becomes the inverse Laplace transform. For a given lattice $G\left(\tau / a_{\tau}\right)$ with finite error bars, there still exist numerous $\rho(\omega)$ 's which satisfy Eq. 3.1. To this problem, Bayes theorem for the conditional probability

$$
P[X \mid Y]=P[Y \mid X] P[X] / P[Y],
$$

is applied where $P[X \mid Y]$ is the probability of observing the event $\mathrm{X}$ given that the event $\mathrm{Y}$ is true. For a given Data $D$ and a prior knowledge $(H)$, the probability for the spectral function $(\rho)$ is

$P[\rho \mid D, H] \propto P[D \mid \rho, H] P[\rho \mid H], P[D \mid \rho, H]=e^{-L}, L=\frac{1}{2} \sum_{i}\left(D_{i}-D_{i}^{\rho}\right)^{2} / \sigma_{i}^{2}, \quad P[\rho \mid H]=e^{-S}, S=S[\rho(\omega), m(\boldsymbol{\omega})]$,

where

$$
P[\rho \mid H]=e^{-S}, \quad S=S[\rho(\omega), m(\omega)] .
$$

Here $S$ is a prior and $m(\omega)$ is a default model. Currently, two different priors, Shannon-Jaynes entropy for $S$

$$
S_{S J}=\alpha \int d \omega\left(\rho-m-\rho \log \left(\frac{\rho}{m}\right)\right)
$$

which is called "Maximum Entropy Method [38] and new prior [39]

$$
S_{B R}=\alpha \int d \omega\left(1-\frac{\rho}{m}+\log \left(\frac{\rho}{m}\right)\right)
$$

are under studies. Since only with infinite number of data points in $G(\tau)$ and zero statistical errors all methods should agree [38], many different priors must be tested with finite number of data points and non-zero statistical errors.

\subsection{Upto last year's conference}
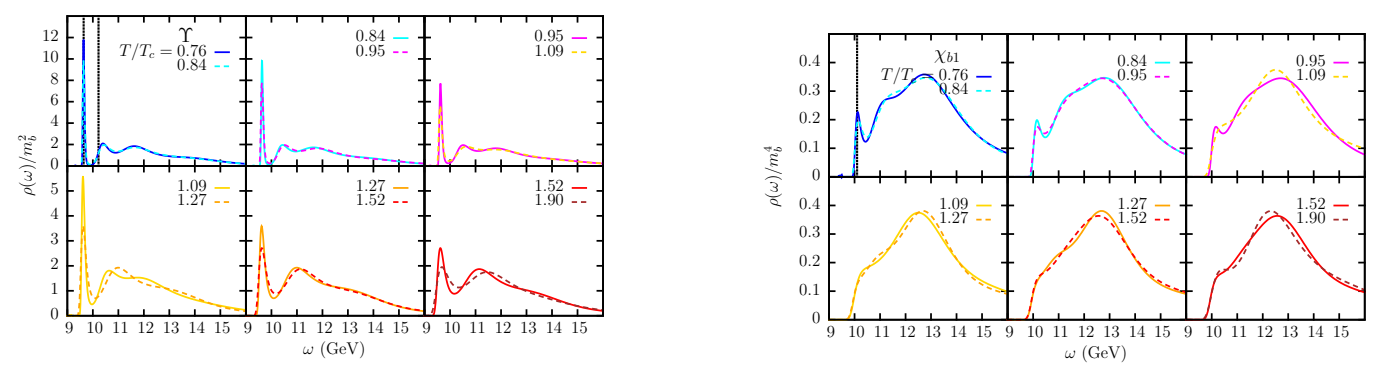

Figure 4: Spectral functions for the $\Upsilon$ channel (left) and those for the $\chi_{b 1}$ channel (right) at successive temperatures from FASTSUM collaboration using NRQCD heavy quark propagator and MEM for the reconstruction of spectral function [18].

Using anisotropic lattices with a fixed lattice scale and temperature change by $N_{\tau}$, FASTSUM collaboration calculated $T \neq 0$ quarkonium correlator using NRQCD and obtained the spectral 
functions of S-wave and P-wave quarkonium correlators using MEM. Using the 1st generation configurations $\left(12^{3} \times N_{\tau}, a_{s} / a_{\tau}=6.0, N_{f}=2\right.$ where $m_{\pi} / m_{\rho} \approx 0.54, T_{c} \approx 210 \mathrm{MeV}$ using Twoplaquette Symanzik Improved gauge action and the fine-Wilson, coarse-Hamber-Wu fermion action with stout-link smearing [29]), FASTSUM collaboration calculated $T \neq 0$ quarkonium correlator [30] using NRQCD and obtained the spectral functions of quarkonium correlators using MEM for S-wave channel [31], moving S-wave channel [32], and P-wave channel [33]. Anisotropic lattices allow a larger number of temporal lattice sites while satisfying $M a_{s} \geq 1$ (for the validity of NRQCD). With a fixed $a_{\tau}$, the zero point energy shift associated with NRQCD formalism needs to be fixed only once.
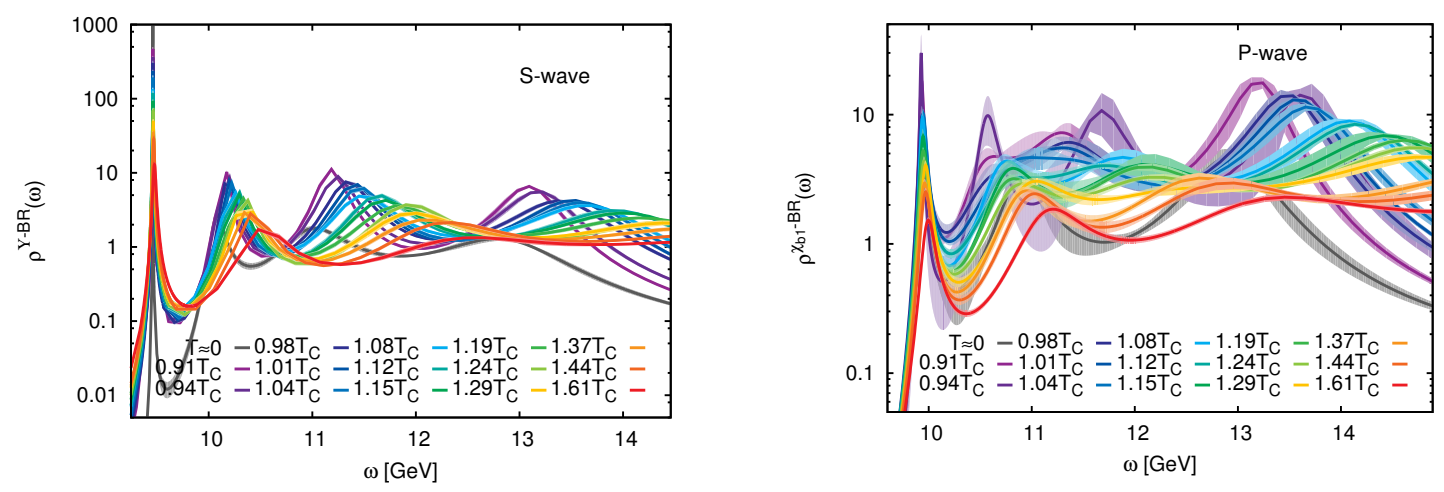

Figure 5: Spectral functions for the $\Upsilon$ channel (left) and those for the $\chi_{b 1}$ channel at successive temperature from [34] using NRQCD heavy quark propagator and new Bayesian prior [39] for the reconstruction of spectral function.

From the spectral function of the $\Upsilon$ channel, it is found that $\Upsilon(1 S)$ survives upto $T \sim 2.1 T_{c}$ while $\Upsilon(2 S)$ and $\Upsilon(3 S)$ are sequentially suppressed in agreement with [2]. $\chi_{b 1}$ melts immediately above $T_{c}$ [33]. A detailed study of systematic errors is performed: default-model dependence in the MEM prior, energy window dependence in the spectral function reconstruction, dependence on the number of configurations and dependence on the Euclidean time window were studied and found that the reconstructed spectral functions are stable. However, with non-zero statistical errors in the data of quarkonium correlators and finite number of the temporal lattice sites, choice of the prior $S$ is not unique and reconstructed spectral functions may depend on the choice. Using the same setup on the 2nd generation configurations $\left(24^{3} \times N_{\tau}, a_{s} / a_{\tau}=3.5, N_{f}=2+1\right)$, FASTSUM collaboration confirmed their earlier conclusions[18]: the survival of $\Upsilon(1 S)$ upto $\sim 1.9 T_{c}$ and the melting of $\chi_{b 1}$ just above $T_{c}$. Fig. 4 shows the spectral functions for the $\Upsilon$ channel and that for the $\chi_{b 1}$ channel.

For a quantitative understanding of the quarkonium melting, FASTSUM's results need to be checked with a setup which has different systematics. Authors of [34] calculated NRQCD heavy quark propagator using a isotropic lattice configurations from HotQCD $\left(48^{3} \times 12, N_{f}=2+1\right.$ light $m_{\pi} \sim 160 \mathrm{MeV}$ and $T_{c}=154 \mathrm{MeV}$ ) [40] where the temperature is changed by changing the lattice spacing $a$ with fixed $N_{\tau}=12$. The pro is that the temperature can be changed continuously and a detailed temperature scan is possible. The con is that the zero energy shift for NRQCD needs to be fixed by accompanying $T=0$ calculations.

Similar to the works by FASTSUM collaboration, a detailed study of systematic errors is also performed. In addition, an investigation on the prior dependence of the reconstructed spectral 
functions by using two different priors, MEM and a new Bayesian prior [39]. [34] found that qualitatively similar behavior for the $\Upsilon$ channel found by FASTSUM: the survival of $\Upsilon(1 S)$ upto the highest temperature they studied $\left(\sim 1.6 T_{c}\right)$ from two different priors. The left figure of Fig. 5 shows the spectral function of the $\Upsilon$ channel obtained with new Bayesian prior. However, qualitatively different behaviors for the $\chi_{b 1}$ channel is found: the spectral function from MEM shows melting but the spectral function from new Bayesian prior shows survival of $\chi_{b 1}$ upto $\sim 1.6 T_{c}$ (the right figure in Fig. 5).

The reconstruction of the spectral function with new Bayesian priors is generally stronger in identifying peaks [41] but is susceptible to spurious "ringing" (i.e., reconstructs peaks for the spectral function from lattice free quarkonium correlators although an analytic result for the spectral function does not show such peaks [34]).

\subsection{Since last year's conference}

Bayesian reconstructions of spectral functions for the correlators with a finite amount of data points and finite statistical errors are expected to show prior dependence. Between MEM prior and new Bayesian prior, which one is closer to the "Bayesian continuum limit" (infinite data points and zero statistical errors) can only be tested by going toward the Bayesian continuum limit.
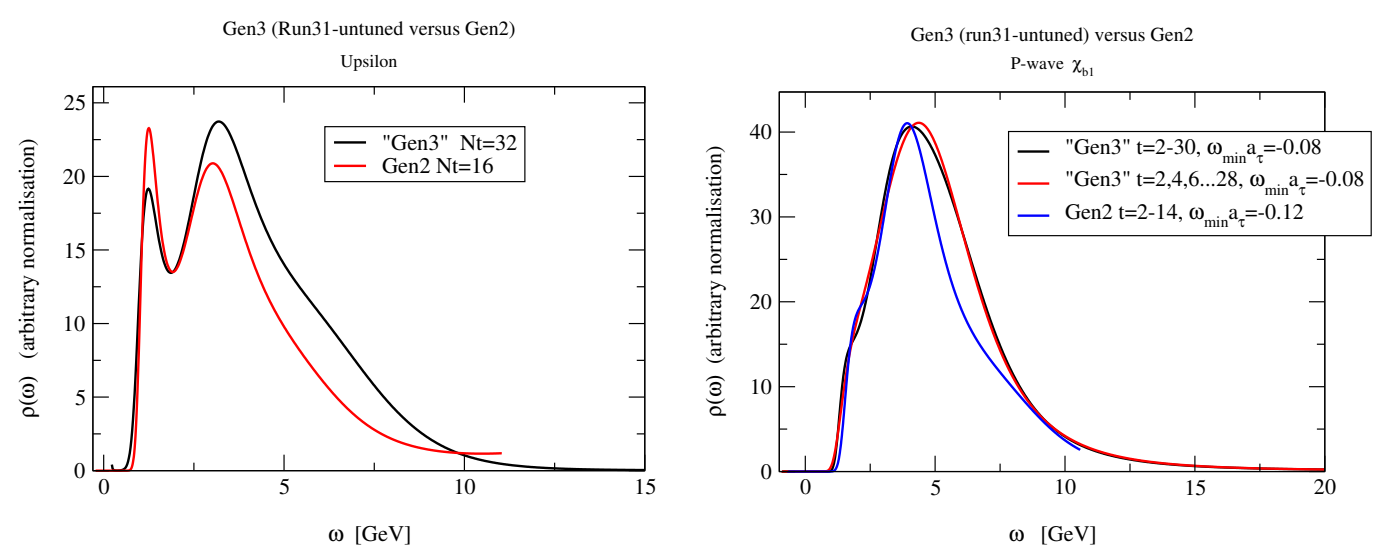

Figure 6: Preliminary spectral functions for the $\Upsilon$ channel (left) and those for the $\chi_{b 1}$ channel (right) from the 3rd Generation configurations (see the text for parameters).

For the further study, FASTSUM collaboration focus on testing a direction of the Bayesian continuum limit by halving the lattice spacing (i.e., increasing the temporal lattice data points by twice) while keeping other conditions the same. In Fig. 6, preliminary results of the spectral functions from the " 3 rd generation configurations $\left(32^{3} \times N_{\tau}, a_{s} / a_{\tau}=6.85, N_{f}=2+1\right)$ show no significant differences between the results from the 2 nd generation configuration and those from the preliminary 3rd generation configurations. On the other hand, authors of [34] concentrate on increasing statistics ( $\sim 4000$ correlators) so that the error bars in the correlators gets small. Also, they explore bottomonium at higher temperatures $\left(T=273\left(1.84 T_{c}\right), 333\left(2.25 T_{c}\right), 407\left(2.75 T_{c}\right)\right.$ using new HotQCD gauge configurations[42]. Fig. 7 show preliminary results of the reconstructed spectral functions of bottomonium correlators at higher statistics and higher temperature. Interestingly, at higher temperature, $\chi_{b 1}$ finally shows melting behavior. 

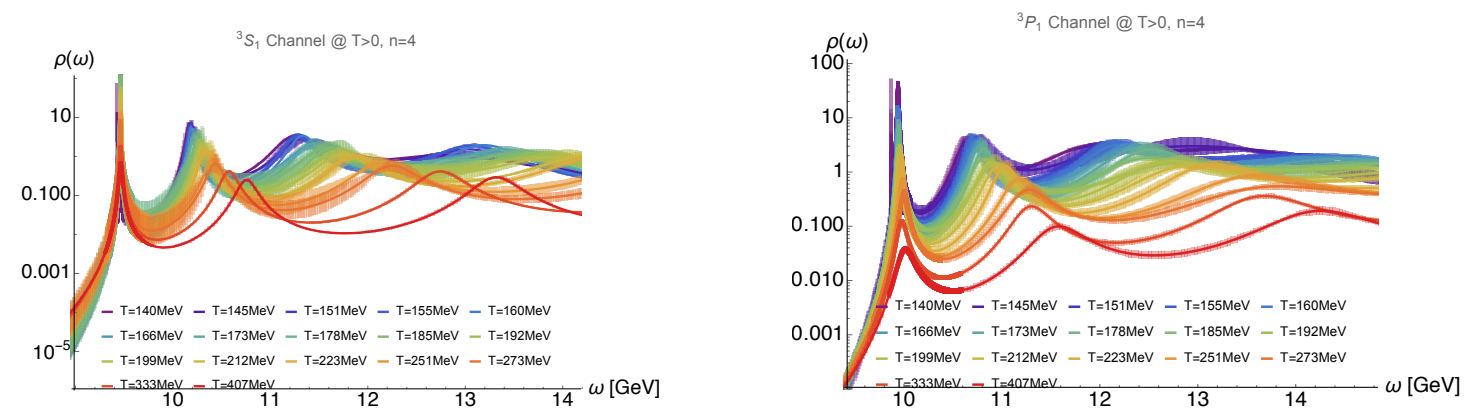

Figure 7: Preliminary spectral functions for the $\Upsilon$ channel (left) and those for the $\chi_{b 1}$ channel (right) from S.K. A. Rothkopf, P. Petreczky with four times higher statistics correlators and higher temperature (see the text for parameters).

\section{Summary}

Some of recent progresses in the lattice studies of heavy flavours in a thermal environment are discussed after looking over the opportunities offered by studying the properties of QGP through heavy quark flavours and the challenges faced in such lattice studies. Focuses in this talk are on the transport phenomena of heavy quarks in thermal medium and the spectral properties of quarkonia in thermal medium.

Various effective field theories for heavy quark and quarkonia on a $T=0$ lattice are well understood and give us many accurate results on the properties of QCD. The same effective field theory on a non-zero temperature lattice alleviates the problem associated with various scales in a heavy quark calculation (lattice spacing, Compton wave length of heavy quark, and large physical size of a lattice) by "integrating out" the momentum scale greater than the heavy quark mass.

In calculating transport properties of heavy quark such as the kinetic equilibriation and the chemical equilibriation, heavy quark mass limit offers a formulation of transport properties as a tail of the spectral function, instead of the usual transport peak of the spectral function as a transport coefficient. Since a transport peak of the heavy quark spectral function is narrow $(\sim$ $\left.\alpha_{s}^{2} T^{2} / M\right)$, this alternative formulation avoids the numerical difficulty of accessing a narrow peak in the study of kinetic equilibriation and also new observable defined in the formulation gives less complicated spectral functions. It gives us a robust determination of the momentum diffusion coefficient for heavy quark at least in the quenched approximation. $\kappa / T^{3}=1.8-3.4$ for charm quark of [16] gives $\mathscr{O}(1) \mathrm{fm} / \mathrm{c}$ as the kinetic equilibriation time scale which is similar to the light parton kinetic equilibriation time scale. Although it is difficult to generalize the same setup to the case of fully dynamical lattices (because the new observable is gluonic), quenched non-perturbative estimate of the momentum diffusion constant is a significant step toward understanding fast kinetic equilibriation of charm quark in relativistic heavy ion collisions.

In the case of heavy quark chemical equilibriation, "tail of a spectral function as a transport coefficient" allows a non-perturbative definition of the thermal averaged Sommerfeld factor (and related $\left.\Gamma_{\text {chem }}\right)$ as a ratio of two-point correlators. This factor is calculable using lattice NRQCD method (and the chemical equilibriation rate, given as the heavy quark number susceptibility times the Sommerfeld factor). Using dynamical $N_{f}=2+1$ lattices $\left(m_{\pi} \simeq 400 \mathrm{MeV}, m_{K} \simeq 500 \mathrm{MeV}\right)$, [9] 
found much larger Sommerfeld enhancement $(\sim \mathscr{O}(100))$ than estimated by a perturbative method. Phenomenologically, at $T \sim 400 \mathrm{MeV}$, for a charm quark mass $M \sim 1.5 \mathrm{GeV}, \Gamma_{\text {chem }}^{-1} \sim 150 \mathrm{fm} / \mathrm{c}$, which suggests that within the lifetime $\sim 10 \mathrm{fm} / \mathrm{c}$ of QGP phase in the current relativistic heavy ion collision experiment, the chemical equilibriation is not achieved. Since the chemical equilibriation rate changes rapid with temperature, however, the planned Future Circular Collider [43] heavy ion program may yield charm quark chemical equilibriation.

Over the years, the lattice community studied thermal behavior of quarkonium by various methods. Recently, using lattice NRQCD to calculate a heavy quark propagator and using Bayesian method to obtain spectral functions of temporal quarkonium correlators constructed from this NRQCD heavy quark propagator overcame various difficulties identified in studying quarkonium on a lattice. Up until this year's lattice conference, two groups which have used this method in different lattice setup reported that the survival of $\Upsilon(1 S)$ upto $\sim 1.9 T_{c}$ ( or1.6T $)$ and sequential suppression of higher $\Upsilon$ states as temperature increases. But the results on $\chi_{b 1}$ states differ: FASTSUM reports the melting of P-wave immediately above $T_{c}$ but the result of [34] hints at the survival of $\chi_{b 1}$ upto $\sim 1.6 T_{c}$. In these studies, quarkonium correlators themselves can be calculated to high accuracy. However, since Bayesian methods can give a unique result only in the Bayesian continuum limit, further studies are needed. FASTSUM group focuses on increasing the data points of temporal quarkonium correlators. Preliminary result shows that doubling the number of the temporal lattice sites does not change significantly the reconstructed spectral functions of $\Upsilon$ and $\chi_{b 1}$. Authors of [34] focus on increasing temperature and increasing statistics. Their preliminary result show that at higher temperature, $\chi_{b 1}$ melts and higher statistics reduces jack-knife errors in the reconstructed spectral functions. More studies by both groups are forthcoming. Also, further investigations on the Bayesian reconstruction method for the spectral functions are needed.

\section{Acknowledgements}

I would like to acknowledge that many discussions with G. Aarts, C. Allton, N. Brambilla, Y. Burnier, A. Francis, O. Kaczmarek, M. Laine, M.P. Lombardo, P. Petreczky and A. Vairo were helpful to my understanding on "heavy flavors in non-zero temperature". This work is supported by the National Research Foundation of Korea under grant No. 2015R1A2A2A01005916 funded by the Korean government (MEST).

\section{References}

[1] B. B. Abelev et al. [ALICE Collaboration], "Azimuthal anisotropy of D meson production in $\mathrm{Pb}-\mathrm{Pb}$ collisions at $\sqrt{s_{\mathrm{NN}}}=2.76$ TeV,” Phys. Rev. C 90 (2014) no.3, 034904 [arXiv:1405.2001 [nucl-ex]].

[2] S. Chatrchyan et al. [CMS Collaboration], "Observation of sequential Upsilon suppression in $\mathrm{PbPb}$ collisions,” Phys. Rev. Lett. 109 (2012) 222301 [arXiv:1208.2826 [nucl-ex]].

[3] G. T. Bodwin, E. Braaten and G. P. Lepage, "Rigorous QCD analysis of inclusive annihilation and production of heavy quarkonium,” Phys. Rev. D 51 (1995) 1125 Erratum: [Phys. Rev. D 55 (1997) 5853] [hep-ph/9407339].

[4] N. Brambilla, A. Pineda, J. Soto and A. Vairo, "Effective field theories for heavy quarkonium," Rev. Mod. Phys. 77 (2005) 1423 [hep-ph/0410047].

[5] N. Isgur and M. B. Wise, "Weak Decays of Heavy Mesons in the Static Quark Approximation," Phys. Lett. B 232 (1989) 113. 
[6] S. Caron-Huot, M. Laine and G. D. Moore, "A Way to estimate the heavy quark thermalization rate from the lattice," JHEP 0904 (2009) 053 [arXiv:0901.1195 [hep-lat]].

[7] D. Bödeker and M. Laine, "Heavy quark chemical equilibration rate as a transport coefficient," JHEP 1207 (2012) 130 [arXiv:1205.4987 [hep-ph]].

[8] D. Bödeker and M. Laine, "Sommerfeld effect in heavy quark chemical equilibration,” JHEP 1301 (2013) 037 [arXiv:1210.6153 [hep-ph]].

[9] S. Kim and M. Laine, "Rapid thermal co-annihilation through bound states in QCD," JHEP 1607 (2016) 143 [arXiv:1602.08105 [hep-ph]].

[10] J.-I. Skullerud and A. Kelly, “Open charm correlators and spectral functions at high temperature," in this proceeding.

[11] A. Ikeda, M. Asakwa, M. Kitazawa, "Charm quark diffusion coefficient from nonzero momentum Euclidean correlator in temperal channel," in this proceeding.

[12] H. Ohno, H.-T. Ding, O. Kaczmarek, S. Mukherjee and H.-T. Shu, "Stochastic reconstruction of charmonium spectral functions at finite temperature," in this proceeding.

[13] H.-T. Shu, H.-T. Ding, O. Kaczmarek, H. Ohno and S. Mukherjee, "Stochastic approaces to extract spectral functions from Euclidean correlators," in this proceeding.

[14] A. Rothkopf and Y. Burnier, "A gauge invariant Debye mass for the complex heavy quark potentia," in this proceeding.

[15] J. I. Kapusta and C. Gale, "Finite-temperature field theory: Principles and applications," Cambridge University Press, Cambridge, U.K. (2006).

[16] A. Francis, O. Kaczmarek, M. Laine, T. Neuhaus and H. Ohno, "Nonperturbative estimate of the heavy quark momentum diffusion coefficient,” Phys. Rev. D 92 (2015) no.11, 116003 [arXiv:1508.04543 [hep-lat]].

[17] A. Sommerfeld, “Über die Beugung und Bremsung der Elektronen,” Ann. Phys. (Leipzig) 403 (1931) 257.

[18] G. Aarts, C. Allton, T. Harris, S. Kim, M. P. Lombardo, S. M. Ryan and J. I. Skullerud, "The bottomonium spectrum at finite temperature from $\mathrm{N}_{f}=2+1$ lattice QCD," JHEP 1407 (2014) 097 [arXiv:1402.6210 [hep-lat]].

[19] T. Matsui and H. Satz, “J/ $\psi$ Suppression by Quark-Gluon Plasma Formation,” Phys. Lett. B 178 (1986) 416.

[20] M. Laine, O. Philipsen, P. Romatschke and M. Tassler, "Real-time static potential in hot QCD," JHEP 0703 (2007) 054 [hep-ph/0611300].

[21] A. Rothkopf, T. Hatsuda and S. Sasaki, "Complex Heavy-Quark Potential at Finite Temperature from Lattice QCD,” Phys. Rev. Lett. 108 (2012) 162001 [arXiv:1108.1579 [hep-lat]].

[22] Y. Burnier and A. Rothkopf, "Disentangling the timescales behind the non-perturbative heavy quark potential,” Phys. Rev. D 86 (2012) 051503 [arXiv:1208.1899 [hep-ph]].

[23] Y. Burnier, O. Kaczmarek and A. Rothkopf, "Static quark-antiquark potential in the quark-gluon plasma from lattice QCD,” Phys. Rev. Lett. 114 (2015) no.8, 082001 [arXiv:1410.2546 [hep-lat]].

[24] Y. Ikeda and H. Iida, "Quark-anti-quark potentials from Nambu-Bethe-Salpeter amplitudes on lattice," Prog. Theor. Phys. 128 (2012) 941 [arXiv:1102.2097 [hep-lat]]. 
[25] H. Iida and Y. Ikeda, "Inter-quark potentials from NBS amplitudes and their applications," PoS LATTICE 2011 (2011) 195.

[26] C. Allton, W. Evans, P. Giudice and J. I. Skullerud, "The Charmonium Potential at Non-Zero Temperature," arXiv:1505.06616 [hep-lat].

[27] Y. Burnier, O. Kaczmarek and A. Rothkopf, "Quarkonium at finite temperature: Towards realistic phenomenology from first principles," JHEP 1512 (2015) 101 [arXiv:1509.07366 [hep-ph]].

[28] Y. Burnier, O. Kaczmarek and A. Rothkopf, "In-medium P-wave quarkonium from the complex lattice QCD potential,” JHEP 1610 (2016) 032 [arXiv:1606.06211 [hep-ph]].

[29] G. Aarts, C. Allton, M. B. Oktay, M. Peardon and J. I. Skullerud, "Charmonium at high temperature in two-flavor QCD," Phys. Rev. D 76 (2007) 094513 [arXiv:0705.2198 [hep-lat]].

[30] G. Aarts, S. Kim, M. P. Lombardo, M. B. Oktay, S. M. Ryan, D. K. Sinclair and J.-I. Skullerud, "Bottomonium above deconfinement in lattice nonrelativistic QCD," Phys. Rev. Lett. 106 (2011) 061602 [arXiv:1010.3725 [hep-lat]].

[31] G. Aarts, C. Allton, S. Kim, M. P. Lombardo, M. B. Oktay, S. M. Ryan, D. K. Sinclair and J. I. Skullerud, "What happens to the Upsilon and eta $a_{b}$ in the quark-gluon plasma? Bottomonium spectral functions from lattice QCD," JHEP 1111 (2011) 103 [arXiv:1109.4496 [hep-lat]].

[32] G. Aarts, C. Allton, S. Kim, M. P. Lombardo, M. B. Oktay, S. M. Ryan, D. K. Sinclair and J. I. Skullerud, "S wave bottomonium states moving in a quark-gluon plasma from lattice NRQCD," JHEP 1303 (2013) 084 [arXiv:1210.2903 [hep-lat]].

[33] G. Aarts, C. Allton, S. Kim, M. P. Lombardo, S. M. Ryan and J.-I. Skullerud, "Melting of P wave bottomonium states in the quark-gluon plasma from lattice NRQCD," JHEP 1312 (2013) 064 [arXiv:1310.5467 [hep-lat]].

[34] S. Kim, P. Petreczky and A. Rothkopf, "Lattice NRQCD study of S- and P-wave bottomonium states in a thermal medium with $N_{f}=2+1$ light flavors," Phys. Rev. D 91 (2015) 054511 [arXiv:1409.3630 [hep-lat]].

[35] G. Aarts and J. M. Martínez Resco, "Transport coefficients, spectral functions and the lattice," JHEP 0204 (2002) 053 [arXiv:hep-ph/0203177].

[36] T. Umeda, "A Constant contribution in meson correlators at finite temperature," Phys. Rev. D 75 (2007) 094502 [hep-lat/0701005].

[37] P. Petreczky, “On temperature dependence of quarkonium correlators," Eur. Phys. J. C 62 (2009) 85 [arXiv:0810.0258 [hep-lat]].

[38] M. Asakawa, T. Hatsuda and Y. Nakahara, "Maximum entropy analysis of the spectral functions in lattice QCD,” Prog. Part. Nucl. Phys. 46 (2001) 459 [hep-lat/0011040].

[39] Y. Burnier and A. Rothkopf, "Bayesian Approach to Spectral Function Reconstruction for Euclidean Quantum Field Theories," Phys. Rev. Lett. 111 (2013) 182003 [arXiv:1307.6106 [hep-lat]].

[40] A. Bazavov et al., "The chiral and deconfinement aspects of the QCD transition," Phys. Rev. D 85 (2012) 054503 [arXiv:1111.1710 [hep-lat]].

[41] Y. Burnier and A. Rothkopf, "Benchmarking the Bayesian reconstruction of the non-perturbative heavy $Q \bar{Q}$ potential," PoS LATTICE 2013 (2014) 491 [arXiv:1310.0165 [hep-lat]].

[42] A. Bazavov et al. [HotQCD Collaboration], "Equation of state in ( 2+1 )-flavor QCD," Phys. Rev. D 90 (2014) 094503 [arXiv:1407.6387 [hep-lat]].

[43] A. Dainese et al., "Heavy ions at the Future Circular Collider," arXiv:1605.01389 [hep-ph]. 